\title{
Anabases
}

ANABASES Traditions et réceptions de l'Antiquité

28 | 2018

Varia

\section{Laurel FULKERSON, Ovid. A Poet on the Margins}

\section{Cyrielle Landrea}

\section{OpenEdition}

Journals

Édition électronique

URL : http://journals.openedition.org/anabases/8205

DOI : 10.4000/anabases.8205

ISSN : 2256-9421

Éditeur

E.R.A.S.M.E.

\section{Édition imprimée}

Date de publication : 9 novembre 2018

Pagination : 375-376

ISSN : 1774-4296

\section{Référence électronique}

Cyrielle Landrea, "Laurel fulkerson, Ovid. A Poet on the Margins », Anabases [En ligne], 28 | 2018, mis en ligne le 09 novembre 2018, consulté le 20 janvier 2021. URL : http://journals.openedition.org/ anabases/8205; DOI : https://doi.org/10.4000/anabases.8205

Ce document a été généré automatiquement le 20 janvier 2021.

(c) Anabases 


\title{
Laurel FULKERSON, Ovid. A Poet on the Margins
}

\author{
Cyrielle Landrea
}

\section{RÉFÉRENCE}

Laurel FULKERSON, Ovid. A Poet on the Margins, Londres, Bloomsbury, 2016, 104 p., 14,99

livres / ISBN 978.1.4725.3134.6.

1 La collection Classical World de Bloomsbury offre de courts ouvrages thématiques sur le monde gréco-romain. Elle s'adresse aux étudiants en début de cursus universitaire, ainsi qu'aux enseignants du secondaire. L'enjeu est d'initier le lecteur avec ces guides accessibles, tout en les invitant à poursuivre leur étude, notamment grâce à une bibliographie thématique (p. 89-92). L'ensemble se décompose en trois chapitres et le choix effectué montre clairement qu'il ne s'agit pas seulement d'une biographie. Dès la préface L. Fulkerson précise bien que le livre concernera plus la poésie que l'homme. Trois chapitres montrent le parti-pris de l'auteur, mettant d'abord en lumière la marginalité d'Ovide dans sa vie (Life on the Margins), puis dans la forme et le fond de ses écrits (Repetition compulsion and Ovidian excess), avant de traiter les rapports entre Rome et l'étranger (Romans at Home and Abroad: Identity and the Colonial Subject).

2 La première partie du premier chapitre (Stranger than Fiction: Poet Exiled Under Suspicious Circumstances!) traite l'exil d'Ovide et ses causes. Sans source contemporaine, il faut alors se fier au poète qui décrit un territoire barbare et hostile, tout en se plaignant de vivre loin de Rome. Or L. Fulkerson rappelle justement que Tomis était alors un centre urbain, loin d'être une « contrée sauvage ».

Le chapitre offre aussi à la page 4 un tableau de la chronologie d'Ovide qui n'est pas toujours simple à fixer, notamment pour son œuvre poétique à cause de nombreux remaniements. D'ailleurs c'est dans ce chapitre que sont présentées les œuvres ovidiennes, dont les Métamorphoses constitueraient le chef d'œuvre. La vie d'Ovide ne s'inscrit pas dans la marginalité uniquement à cause de sa relégation ; en effet dans la 
partie suivante (Meanwhile, Back in Rome...: Ovid's Historical Context), l'auteur soulève aussi une frontière chronologique. En effet le poète vécut à un moment d'intenses transformations et d'incertitudes entre une république finissante et l'émergence du principat augustéen tiraillé entre les innovations et le respect des traditions.

Se pose ensuite la question du patronat et des cercles littéraires. Selon l'auteur, Ovide n'aurait pas eu de patron, rappelant seulement ses liens avec Messalla, en fait M. Valerius Messalla Corvinus (cos. 31 av. J.-C.), l'un des personnages les plus importants de l'époque. Le patricien n'aurait alors pas été son patron, mais juste un de ses soutiens les plus importants. La conclusion du chapitre compare Ovide à Auguste : "Ovid, then, is similar to Augustus, as well as serving as his negative mirror reflection » (p. 26). Ils ont effectivement tous les deux transformé le monde romain, le premier par les genres littéraires, l'autre par la mise en place d'un nouveau régime.

5 Le deuxième chapitre (Repetition compulsion and Ovidian excess) caractérise l'aspect littéraire de l'œuvre ovidienne, tant sur le fond que sur la forme. L'attention porte d'abord sur la métrique, le style (qualifié de « straightforward style » à la page 32) et les narrateurs dans la première partie (Now you see him, now you still see him: Ovidian narrative style and metre). Le poète a surtout utilisé la métrique élégiaque. Toutefois l'idée de frontière réapparaît dès la page 30 : «Ovid regularly draws attention to the fact of his almost-but-not-quite status as an epic poet». Concernant les narrateurs, ils posent souvent problème (par exemple par des commentaires hors-de-propos) et ils sont intrusifs, ayant pour conséquence de déstabiliser le lecteur; même constat de déstabilisation pour des faits décrits comme autobiographiques, mais qui ne le sont pas forcément. Puis la seconde partie (Same Story, Different Day) s'attache aux répétitions dans l'œuvre ovidienne sous la forme de reprises - par exemple de Virgile - ou de simples répétitions, voire de révisions de ses propres écrits. Enfin l'auteur traite la réception de l'œuvre ovidienne, réutilisée par Pétrarque notamment.

La première partie du troisième chapitre (Romans at Home and Abroad: Identity and the Colonial Subject) s'intitule Strangers in a Strange Land: Explorers and Exiles. Ces figures en marge (explorateurs, réfugiés et des exilés comme lui) sont à la fois à l'intérieur et à l'extérieur d'une société ou d'une culture. Cette position d'interface est idéale pour une meilleure compréhension du monde. Dans Speaking and silence: Victims and Victimizers, L. Fulkerson s'intéresse ensuite aux rapports oppresseurs/victimes qui, dans la poésie ovidienne, sont loin d'être figés, car les victimes peuvent se transformer en persécuteurs. L'accent est aussi mis sur les femmes. Enfin le dernier thème traite de l'Empire et du colonialisme, interrogeant la romanité d'Ovide, tout en cherchant à savoir s'il était un partisan ou un opposant à Auguste.

Pour conclure, l'ensemble facilite la compréhension de la civilisation romaine à travers la biographie d'Ovide. Par exemple un glossaire des termes latins et des noms propres est inséré à la fin de l'ouvrage (p.93-99), ainsi qu'un index (p.101-104). Certes l'initiation du lecteur implique une grande simplification, mais elle est parfois extrême, car César n'est alors plus qu'un des « warlords of the late Republic » (p. 95). En outre les dates, de naissance et de mort ne sont pas données, ce qui ne permet pas au lecteur de situer ces personnages dans la chronologie de la période (sauf étonnamment pour Livie). Des illustrations sont aussi présentes, notamment une page de manuscrit de l'Ars amatoria (p.10), une mosaïque (p.34) ou un tableau sans référence précise (p.37). 
L'ouvrage remplit cependant sa mission d'initiation, en décrivant la complexité ovidienne; un poète pluriel et présenté comme l'«ideal combination of fox and hedgehog » (préface).

\section{AUTEUR}

\section{CYRIELLE LANDREA}

Université Lille-SHS

cyrielle.landrea@orange.fr 